\title{
Correlation of Nutritional Status and Peak Expiratory Flow Rate in Normal South Indian Children Aged 6 to 10 Years
}

\author{
Sudha. D ${ }^{1}$, Chandra Selvi. E ${ }^{2}$, Saikumar. $\mathrm{P}^{3}$ \\ ${ }^{1,2,3}$ Department of physiology, Sree Balaji Medical College \& Hospital, Bharath University, India.
}

\begin{abstract}
BACKGROUND: Many studies have been proved the relation between the nutritional habit and respiratory functions in south Indian children. The increase in respiratory problems due to increase in Body Mass Index (BMI) in children has been a major worldwide problem. Deposition of fat over the chest wall alters the Peak Expiratory Flow Rate (PEFR) among pulmonary function test parameters. Among all the pulmonary function test parameters Peak expiratory flow rate (PEFR) directly indicate the nutritional status which is also an easy and non invasive method for estimating the lung function in children. AIM: To assess the effect of Body Mass Index (BMI) and Skin Fold Thickness (SFT) on PEFR in children aged 6 to 10 years of both the sexes. METHODS: We recruited 258 children aged 6 to 10 years of both sexes for our study. The anthropometric parameters including height, weight and BMI were measured. PEFR was measured using Peak Expiratory Flow Meter. The skin fold thickness was measured by using Digital skin fold caliper. Results were statistically analyzed by using Pearson Correlation co efficient test and ANOVA. RESULTS: In our study as age progresses the height of the children in both sexes also increases. Our results showed positive correlation between height, BMI and SFT with PEFR. Our results also showed as increase in age BMI remains constant however SFT showed positive correlation in our study population. CONCLUSION: We concluded that among the factors determine the nutritional status including BMI, effect of SFT on PEFR play an important role in the maintenance of normal pulmonary functions in children. Thus our study proved that the reason for the respiratory problem may be due to abnormal SFT which determines the total body fat apart from BMI.
\end{abstract}

Keywords: Peak expiratory flow rate, Body mass index, Skin fold thickness, Total Body Fat.

\section{Introduction}

Body Mass Index (BMI) indicates nutritional status in children either increase or decrease in BMI lead to many problems. The association of higher BMI with lower PEFR may indicate that obesity is an important risk factor for reduced airflow or lung function in children (1). Obese children are at increased risk of obstructive sleep disordered breathing (OSDB)(2). More over in obese children there is an excessive day time sleepiness (EDS), elevation of proinflammatory cytokines, increased leptin and decreased adiponectin. All this changes point to an inflammatory, insulin resistance state in future leads to type 2 Diabetes Mellitus(3). Ehab Dayyat(4), concluded that Obstructive Apnea-Hypopnea Index (OAHI) is more likely to be smaller in obese children compared to non obese children. He also suggested that soft tissue changes and potentially fat deposition in the upper airway may play a significant role in the global differences in tonsillar and adenoidal size among the obese and non obese children with Obstructive sleep apnea (OSA). Many of the well reported respiratory problems associated with Body mass index, have been considered to be diseases of adults are now also affecting children.

According to WHO, children aged around 6 to 10 years either increase or decrease in BMI they develop many respiratory problems. The World Health Organization has described obesity as one of today's most neglected public health problem, affecting every region of the world(5) . India is the second most popular country in the world that contributes to $\sim 17 \%$ of the world's population and contributes to $16 \%$ of the world's death. Nutritional status of the Indian population varies significantly across the regions. Certain regions are associated with extremely high ratio of childhood under nutrition (ranging from $20 \%$ to $80 \%$ ), whereas others have a high prevalence of adult under nutrition $(50 \%)$, and some have both ${ }^{(5)}$.Nowadays food intake in children mainly at the age of 6 to 10 years become altered, may be due to the socioeconomically variation, stress, improper breakfast.

Prevalence of Respiratory diseases in children due to obesity is now recognized as an important risk factor in the development of several Respiratory diseases. Of these Respiratory diseases, it has already been well established that obesity can lead to Obstructive Sleep Apnea (OSA) and Obesity -Hypoventilation Syndrome (OHS). Epidemiological studies have reported that obesity is a risk factor for the development of asthma. Furthermore a number of studies indicated that obesity is also associated with a higher risk of developing deep vein thrombi, pulmonary emboli, pulmonary hypertension and pneumonia. 
There have been only limited studies in children without asthmatic symptoms. Aim of this study is to investigate the relationship between Body Mass Index (BMI) and Peak Expiratory Flow Rate (PEFR) values in children of both sexes between the age group of 6 to 10 years.

\section{Study population:}

\section{Materials And Methods:}

For this study, we recruited the children of age group 6 to 10 years of both sex from the relatives of patients attended the outpatient Department of Pediatrics, Sree Balaji Medical College and Hospital, from the primary schools in and around the Sree Balaji Medical College and Hospital. We recruited a total number of 258 children of age group 6-10 year, in those $156(60.5 \%)$ boys and $102(39.5 \%)$ girls. This study was approved by the institutional ethical committee. Informed consent was obtained from the parents of children who were participated in this study. The procedure to be performed was explained in detail in the local language to each child and their parents.

\section{Exclusion Criteria :}

1. Bronchial Asthma.

2. Acute or Chronic Respiratory Tract Infection (inclusion atleast 3 months before testing).

3. Acute or Chronic Cardiac disease.

4. Any Systemic illness.

5. Structural deformity of the thoracic cage.

\section{Methods:}

All tests were carried out in the morning after breakfast. For each child detailed History and Physical Examination was done. The Anthropometric parameters like height in centimeters, weight in kilo grams were measured. These parameters were used to calculate BSA and BMI. Body mass index (BMI) was calculated using this formula. $\mathrm{BMI}=$ weight in $\mathrm{kg} /$ height in $\mathrm{m}^{2}(6)$. Body surface area (BSA) was calculated using this formula. $\mathrm{BSA}=$ weight $(\mathrm{kg})^{\wedge} 0.425 \times$ height $(\mathrm{cm})^{\wedge} 0.725 \times 71.84 / 10000(7)$.

\section{Peak Expiratory Flow Rate:}

The Predicted PEFR values for children at this age group 6 to 10 years was calculated by using this formula. Predicted value $=$ constant + standing height in $\mathrm{cm} \times$ height coefficient + weight in $\mathrm{kg} \times$ weight coefficient (8). Peak expiratory flow rate was recorded by using peak expiratory flow meter. Before recording the procedure was demonstrated to each child. The subjects were asked to take a deep inspiration until he/she breathed in total lung capacity. Immediately he/she should wrap the mouth tightly around a mouth piece, and asked to breathe out to the maximum effort. The subjects were encouraged to put maximum effort while doing this maneuver. Three such readings were obtained from each child. The highest values obtained were taken as Peak Expiratory Flow Rate (PEFR).

\section{Skin Fold Thickness:}

An efficient and practical way to measure body fat is by measuring skin fold. Skin fold thickness of children was measured by using Digital Skin fold Caliper. The measurements were taken at six sites of the body including biceps, triceps, subscapular, suprailiac, knee and calf. These values were entered in the tabular column of both boys and girls of different ages.

\section{Statistical Analysis:}

The entire data were tabulated and the values were expressed in mean \pm standard deviation. The correlation between PEFR (Measured) and BMI of boys and girls were analyzed by using Pearson Correlation analysis, similarly ANOVA was used to compare within the groups.

\section{Results :}

Table-1. Physical characteristic features of the children $(n=258)$.

\begin{tabular}{|c|c|c|c|c|c|c|c|c|}
\hline & \multicolumn{4}{|c|}{ HEIGHT(cm) } & \multicolumn{4}{c|}{ WEIGHT(kg) } \\
\hline Age in yrs & No & BOYS & N0 & GIRLS & N0 & BOYS & No & GIRLS \\
\hline 6 & 38 & $112.19 \pm 6.6$ & 27 & $110.39 \pm 5.41$ & 38 & $17.17 \pm 3.86$ & 27 & $18.06 \pm 4.92$ \\
\hline 7 & 29 & $117.54 \pm 5.45$ & 11 & $120.54 \pm 7.17$ & 29 & $20.88 \pm 5.72$ & 11 & $26.21 \pm 8.61$ \\
\hline 8 & 39 & $125.36 \pm 8.12$ & 26 & $126.54 \pm 7.07$ & 39 & $24.05 \pm 7.45$ & 26 & $22.65 \pm 6.74$ \\
\hline 9 & 30 & $128.43 \pm 4.48$ & 22 & $129.68 \pm 5.94$ & 30 & $25.3 \pm 3.08$ & 22 & $28.09 \pm 6.3$ \\
\hline 10 & 20 & $134.65 \pm 5.58$ & 16 & $135.75 \pm 5.07$ & 20 & $30.15 \pm 8.6$ & 16 & $31.69 \pm 7.21$ \\
\hline
\end{tabular}


Values are expressed in mean \pm standard deviation.

Table -2. Anthropometric parameters of the children $(n=258)$.

\begin{tabular}{|c|c|c|c|c|c|c|c|c|}
\hline \multirow{2}{*}{$\begin{array}{c}\text { AGE } \\
\text { in yrs }\end{array}$} & \multicolumn{4}{|c|}{ BMI $\left(\mathrm{kg} / \mathrm{m}^{2}\right)$} & \multicolumn{4}{c|}{ BSA $\left(\mathrm{m}^{2}\right)$} \\
\hline & & BOYS & No & GIRLS & No & BOYS & No & GIRLS \\
\hline 6 & 38 & $13.22 \pm 1.90$ & 27 & $14.27 \pm 3.15$ & 38 & $0.718 \pm 0.09$ & 27 & $0.72 \pm 0.10$ \\
\hline 7 & 29 & $14.54 \pm 3.03$ & 11 & $16.14 \pm 3.75$ & 29 & $0.8 \pm 0.12$ & 11 & $0.87 \pm 0.17$ \\
\hline 8 & 39 & $14.84 \pm 1.86$ & 26 & $16.98 \pm 3.32$ & 39 & $0.9 \pm 0.14$ & 26 & $0.94 \pm 0.13$ \\
\hline 9 & 30 & $14.92 \pm 1.86$ & 22 & $18.67 \pm 5.31$ & 30 & $0.93 \pm 0.06$ & 22 & $0.97 \pm 0.12$ \\
\hline 10 & 20 & $15.91 \pm 4.12$ & 16 & $16.35 \pm 3.71$ & 20 & $1.02 \pm 0.15$ & 16 & $1.03 \pm 0.12$ \\
\hline
\end{tabular}

BMI - Body Mass Index BSA - Body Surface Area Values are expressed in mean \pm standard deviation.

As shown in table-1 the height and weight of the children are found to be matched in both sexes in our results. Body mass index (BMI) of the boys at the age of 6, 7, 9, 10 were lower than the girls however BMI of the boys at the age of 9 was statistically significant than the girls which is shown in table-2. The Body surface area (BSA) in all the age group (6 to 10 years) remain the same in both the sex in our study group.

Table - 3. Peak Expiratory Flow Rate in study population $(n=258)$.

\begin{tabular}{|c|c|c|c|c|c|c|c|c|}
\hline \multirow{2}{*}{$\begin{array}{l}\text { AGE } \\
\text { in } \\
\text { yrs }\end{array}$} & \multicolumn{4}{|c|}{ PEFR(Measured) } & \multicolumn{4}{c|}{ PEFR(Predicted) } \\
\cline { 2 - 9 } & No & BOYS & No & GIRLS & No & BOYS & No & GIRLS \\
\hline 6 & 38 & $132.11 \pm 26.73$ & 27 & $109.26 \pm 26.30$ & 38 & $101.65 \pm 8.45$ & 27 & $94.70 \pm 7.39$ \\
\hline 7 & 29 & $160.69 \pm 34.53$ & 11 & $170 \pm 33.17$ & 29 & $160.69 \pm 34.53$ & 11 & $105.45 \pm 12.24$ \\
\hline 8 & 39 & $172.05 \pm 37.15$ & 26 & $138.46 \pm 32.33$ & 39 & $118.53 \pm 11.95$ & 26 & $105.79 \pm 9.95$ \\
\hline 9 & 30 & $195.33 \pm 37.02$ & 22 & $183.64 \pm 41.12$ & 30 & $121.11 \pm 5.25$ & 22 & $116.06 \pm 9.16$ \\
\hline 10 & 20 & $220 \pm 30.44$ & 16 & $191.25 \pm 41.45$ & 20 & $128.54 \pm 10.75$ & 16 & $121.43 \pm 8.06$ \\
\hline \hline
\end{tabular}

PEFR - Peak Expiratory Flow Rate.

Table - 4. Skin fold thickness and Total body fat in study population $(\mathrm{n}=258)$.

\begin{tabular}{|c|c|c|c|c|}
\hline \multirow{2}{*}{$\begin{array}{c}\text { AGE } \\
\text { in } \\
\text { yrs }\end{array}$} & No & BOYS & No & GIRLS \\
\cline { 2 - 5 } & 38 & $31.18 \pm 10.27$ & 27 & $38.12 \pm 15.55$ \\
\hline 6 & $36.89 \pm 7.41$ & 11 & $47.24 \pm 17.33$ \\
\hline 7 & 29 & $49.77 \pm 11.75$ & 26 & $46.87 \pm 16.64$ \\
\hline 8 & 39 & $58.39 \pm 14.48$ & 22 & $81.85 \pm 16.56$ \\
\hline 9 & 30 & $72.13 \pm 18.76$ & 16 & $89.03 \pm 15.08$ \\
\hline 10 & 20 & \multicolumn{3}{|c}{} \\
\hline \hline
\end{tabular}

Skin fold thickness and Total body fat - values are expressed in mean \pm standard deviation.

The Peak expiratory flow rate (PEFR-Measured) of the boys at the age of $6,8,9,10$ were higher than the girls however PEFR of the boys at the age of 7 was not statistically significant than the girls as shown in table- 3. The Peak expiratory flow rate (Predicted) of the boys at the age of 6, 7, 8, 9, 10 were higher than the girls however PEFR of the boys at the age of 8, 9, 10 were not statistically significant. Skin fold thickness (SFT) of the boys at the age of $6,7,9,10$ were lower than the girls however SFT of the boys at the age of 8 was not statistically significant as shown in table-4. 
Fig: 1. Correlation between height and PEFR at the age $6-10$ years of both sexes.
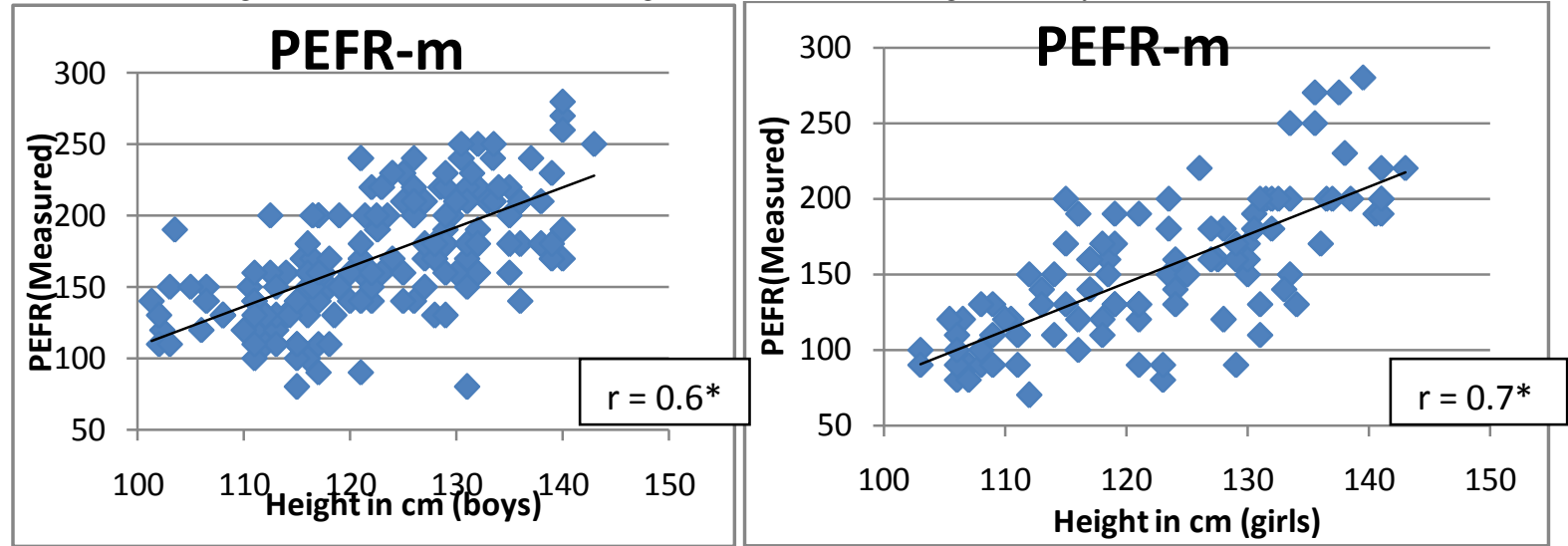

Fig: 2. Correlation between BMI and SFT at the age of 6 to 10 years in both sexes.
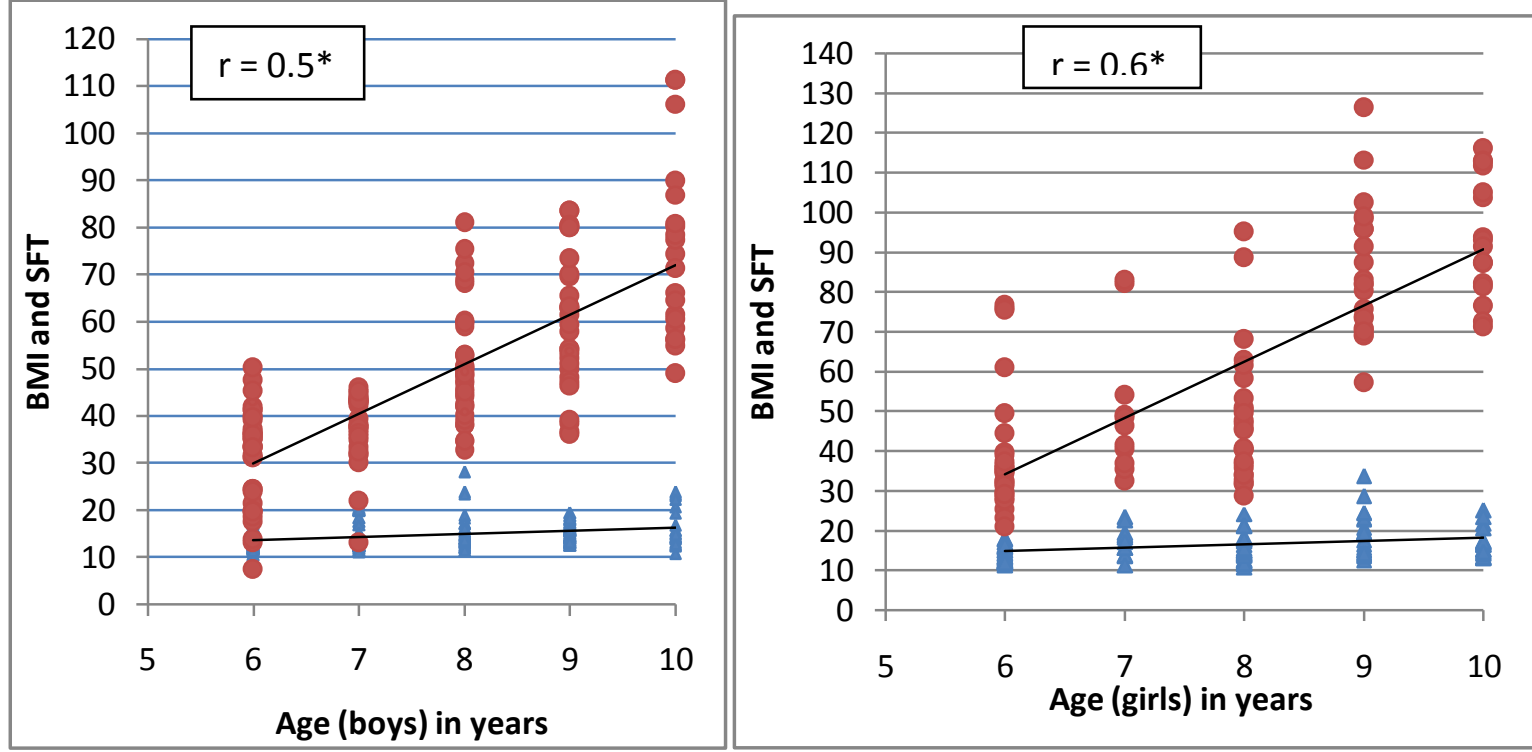

Fig: 3. Correlation between SFT and PEFR (Measured) at the age of 6 to 10 years in both sexes.
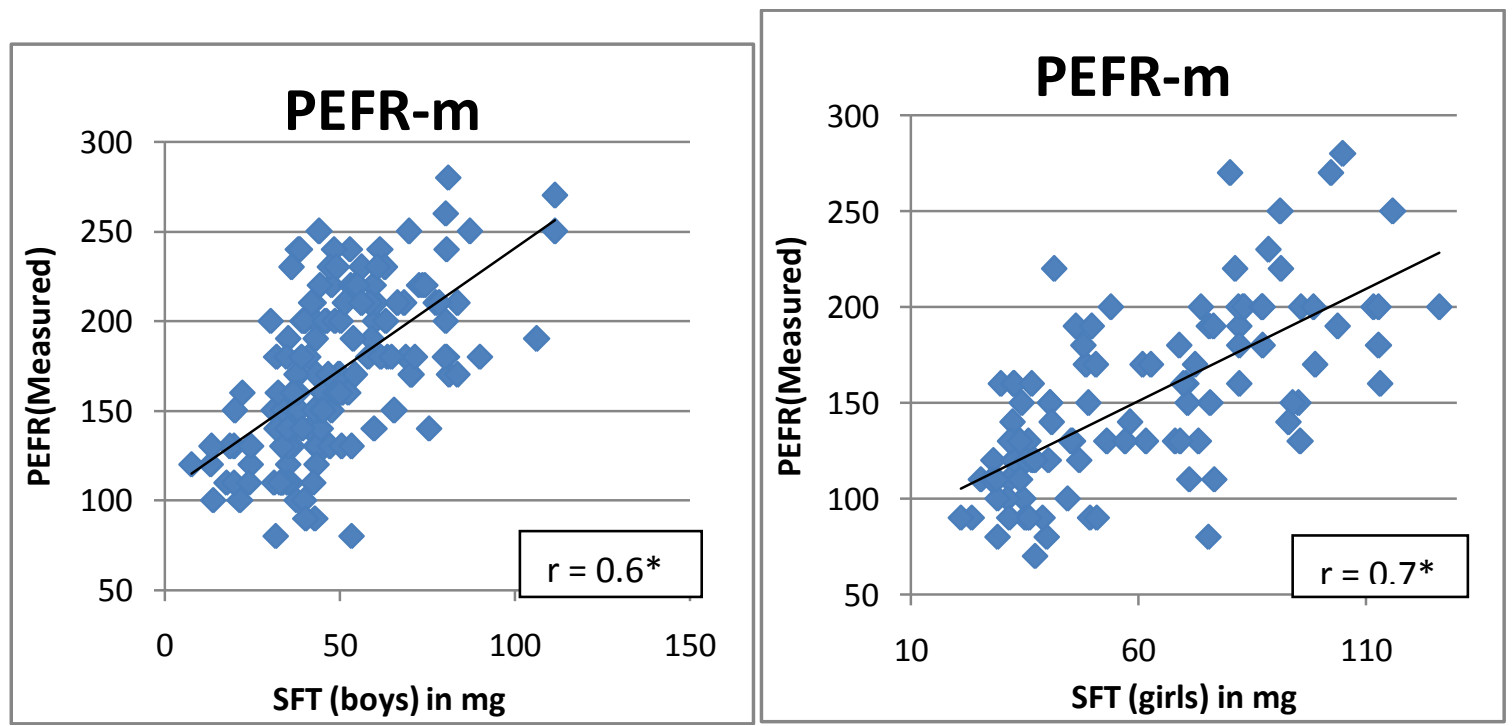

Fig: 4. PEFR (Measured) of boys and girls at the age of 6 to 10 years. 


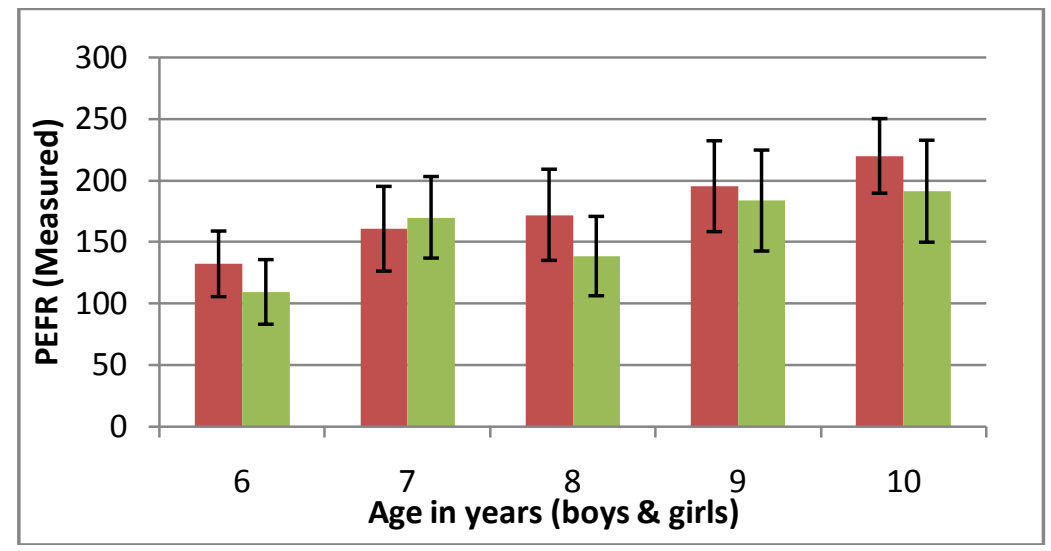

Fig: 1 shown the PEFR and height at the age of 6 to 10 years in both sex. Boys $\left(r=0.3^{*}\right)$ and girls $\left(r=0.6^{*}\right)$. PEFR (Measured) and height were positively correlated which has statistically significant. Fig: 2 shown the BMI and SFT at the age of 6 to 10 years in both sexes. Boys $\left(r=0.5^{*}\right)$ and girls $\left(0.6^{*}\right)$. BMI and SFT were positively correlated which has statistically significant. Fig: 3 shown the PEFR (Measured) and SFT at the age of 6 to 10 years in both sexes. Boys $\left(r=0.6^{*}\right)$ and girls $\left(0.7^{*}\right)$. PEFR (Measured) and SFT were positively correlated which has statistically significant.

\section{Discussion}

The physical characteristics of height and weight of the children (both sexes) at the age of 6 to 10 years and their variations were given in table 1.The height and weight consistently increased with age of the children as revealed by vijayan et al(8) in south Indian children. But our study showed at the age of 6 to 10 years when compared to boys, height and weight of the girls significantly increased in their values. This may be due to gender differences in nutritional status in between boys and girls at the age of 6 to 10 years may influence this variation. However in contrast to our study many studies stated that nutritional status appeared to be gender neutral only in the South(9).Furthermore it has been assumed that earlier puberty in girls was the reason for increase in weight and height of the girls when compared to the boys.

The observations for BMI and BSA were summarized in table 2. The results of analysis of variance provided evidence of significant $(\mathrm{P}<0.000)$ interaction, thus indicating that BMI and BSA were not independent in their influence on their age and sex (9). Our result showed increase in BMI and BSA in both the sexes which was consistence with age. However the BMI and BSA of the boys showed lesser values than the girls. The reason may be due to difference in distribution of fat and physical activity among the gender.

As shown in previous studies height being an important factor for determining the PEFR, height was significantly correlated with PEFR in our study in both sexes. The PEFR (Measured) values increased in linear relation to age and height at the age of 6 to 10 years. Our study also showed that height influences the prediction equation in males to a greater extent whereas weight and skin fold thickness had greater influence in girls. Wang et al (10), concluded that for the same height boys have greater lung function values than girls. Similar to our study Chowgule et al (11) measured PEFR in children age between 6 to10 years. The PEFR variables showed a linear positive correlation with height and age. Boys show higher values for lung function variables than girls. Height proved the maximum variations in PEFR. These results were similar to the findings of our study. As age, height, weight, BMI and BSA have all been used in studies either alone or in combination to predict PEFR (12$15)$.

The present study reported that PEFR have shown significant correlation with BMI. Similar findings were reported by some authors (16-17).The PEFR (Predicted) values as per the height of the children are lesser than the PEFR(Measured) values. This indicates that height determines the PEFR (Predicted) values but BMI and SFT also has an influence in PEFR (Measured) values. Height is indirectly proportionate with BMI and BSA. Children having lesser BMI and SFT, the PEFR (Measured) values were higher. In our study the boys had lesser values of BMI and SFT than the girls as the result of it the PEFR (Measured) values of the boys were higher than the girls.

Skin fold thickness (SFT) is also one of the parameter which influences the PEFR (Measured) values of the children. Since more accumulation of fat reduces the muscle strength and there is a slight decrease effort in stretching of respiratory muscle. The reason for this is may be due decreased physical activity and exercise, traditional customs, modified sedentary life style and increased intake of junk foods influence in deposition of more fat. As shown in table 4 there was a steady increase in SFT in both sexes as age progresses from 6 to 10 years. SFT is inversely proportionate to the PEFR (Measured) values. The girls had higher SFT values than boys as a result the PEFR (Measured) values of the girls were lesser than the boys. 


\section{Conclusion}

Our study proved the positive correlation between BMI, SFT with PEFR. So we concluded that excessive deposition of fat in children is associated with the decrease in PEFR values. It is necessary to have a good physical activity in children to avoid the future respiratory problems. In order to ensure health of the children the simple and low-cost suggestion, such as yoga, meditation and folk dance, can be used to maintain optimal weight of up coming generation.

\section{Reference:}

[1] Gundogdu Z, Eryilmaz. N. Correlation between peak flow and body mass index in obese and non obese children. Prim Care Re spir J. 2011; 20: 403-6.

[2] Y Wing, S Hui, W Pak, C Ho, A Cheung, A Li and T Fok. A controlled study of sleep related disordered breathing in obese children. Arch Dis Child. 2003; 88: 1043-1047.

[3] Marina Tsaoussoglou, Edward O. Bixler, Susan Calhoun, George P.Chrousos, K. Sander, and Alexandros N. Vgontzas. J Clin Endocrinol Metab. 2010; 95: 143-150.

[4] Ehab Dayyat, Leila Kheirandish- Gozal, Oscar Sans Capdevila, Muna M. A. Maarafeya, David Gozal. Obstructive Sleep Apnea in children. Chest. 2009; 136: 137-144.

[5] Pednekar MS. Association of body mass index with all cause and cause-specific mortality. Int J Epidemiol 2008; 37:524-35.

[6] Shah C, Diwan J, Rao P, Bhabhor M, Gokhle P, Mehta H, Assessment of obesity in school children. Calcicut Medical Journal 2008; 6:2.

[7] Piyush M.Srivastava, Paul Calafiore, Richard J.Macisaac, Sheilak.Patel, Merlin C.Thomas, George Jerums and Loums M, Burrell.Prevalence and predictors of cardiac hypertrophy and dysfunction in patients with type2 Diabetes. Clinical science 2008; 114: 313-320.

[8] V.K.Vijian, A.M.Reetha, K.V.Kuppurao, P.Venkatesan and S.Thilakavathy, Pulmonary function in normal south Indian children aged 7 to 19 years. Indian J Chest Dis Allied Sci 2000; 42: 147-156.

[9] Alessandro Tarozzi, Aprajit Mahajan. Child Nutrition in India in the Ninetie: A Story of Increased Gender Inequality?; 2005: JEL: I12, J13, O53.

[10] Wang X, Dockery DW, Wypiji D, Fay ME, Ferris BG Jr. Pulmonary function between 6 and 18 years of age. Pediatr pulmonol 1993; 15: 75-88.

[11] Chowgule RV, Shetye VM, Parmar JR. Lung function tests in normal Indian children.Indian Paediatr 1995; 32: 185-191.

[12] Paramesh H.Normal peak expiratory flow rate in urban and rural children. Indian J Pediatr 2003; 70: 375-377.

[13] Swaminathan S, Diffey B, Vaz M. Evaluating the suitability of prediction equations for lung function in Indian children: a practical approach. Indian Pediatr 2006; 43: 680-698.

[14] Prasad R, Verma SK, Agrawal GG, Mathur N. Prediction model for peak expiratory flow in North Indian population. Indian J Chest Dis Allied Sci 2006; 48: 103-106.

[15] Nku CO, Peters EJ, Eshiet AI, Bisong SA, Osim EE. Prediction formulae for lung function parameters in females of south eastern Nigeria. Niger J Physiol Sci 2006; 21:43-47.

[16] Taksande A, Jain M, Vilhekar K, Chaturvedi P. Peak expiratory flow rate of rural school children from Wardha district, Maharashtra in India. World J Pediatr 2008; 4: 211-4.

[17] Debray P, Shreevastsa BM, MG RB, Sen TK, Roy S, Saha CG. A comparative study of the peak expiratory flow rate of Indian and Nepalese young adults in a teaching institute. JNMA J Nepal Med Assoc 2008; 47: 7-11. 